\title{
Probing properties of hot and dense QCD matter with heavy flavor in the PHENIX experiment at RHIC
}

\author{
Rachid Nouicer ${ }^{1}$, on behalf of the PHENIX Collaboration \\ ${ }^{1}$ Department of Physics, Brookhaven National Laboratory, Upton, NY 11973, United States
}

\begin{abstract}
Hadrons carrying heavy quarks, i.e. charm or bottom, are important probes of the hot and dense medium created in relativistic heavy ion collisions. Heavy quarkantiquark pairs are mainly produced in initial hard scattering processes of partons. While some of the produced pairs form bound quarkonia, the vast majority hadronize into particles carrying open heavy flavor. Heavy quark production has been studied by the PHENIX experiment at RHIC via measurements of single leptons from semi-leptonic decays in both the electron channel at mid-rapidity and in the muon channel at forward rapidity. A large suppression and azimuthal anisotropy of single electrons have been observed in $\mathrm{Au}+\mathrm{Au}$ collisions at $200 \mathrm{GeV}$. These results suggest a large energy loss and flow of heavy quarks in the hot, dense matter. The PHENIX experiment has also measured $J / \psi$ production at $200 \mathrm{GeV}$ in $p+p, d+\mathrm{Au}, \mathrm{Cu}+\mathrm{Cu}$ and $\mathrm{Au}+\mathrm{Au}$ collisions, both at mid- and forward-rapidities, and additionally $\mathrm{Cu}+\mathrm{Au}$ and $U+U$ at forward-rapidities. In the most energetic collisions, more suppression is observed at forward rapidity than at central rapidity. This can be interpreted either as a sign of quark recombination, or as a hint of additional cold nuclear matter effects. The centrality dependence of nuclear modification factor, $R_{A A}\left(p_{\mathrm{T}}\right)$, for $J / \psi$ in $U+U$ collisions at $\sqrt{s_{N N}}=193 \mathrm{GeV}$ shows a similar trend to the lighter systems, $\mathrm{Au}+\mathrm{Au}$ and $\mathrm{Cu}+\mathrm{Cu}$, at similar energy $200 \mathrm{GeV}$.
\end{abstract}

\section{Introduction}

The measurement of inclusive hadron yields in central Au+Au collisions at RHIC led to the discovery of the suppression of hadron production at large transverse momenta $\left(p_{T}\right)$ compared to $p+p$ collisions [1]. This is generally attributed to the energy loss of light partons in the dense nuclear matter created at RHIC. Heavy quarks, i.e. charm and beauty, are believed to be mostly created in initial hard scattering processes of partons [2] and thus are excellent probes of the hot and dense matter formed in nucleus-nucleus collisions at high energy. While some of the produced pairs form bound quarkonia, the vast majority hadronize into hadrons carrying open heavy flavor. They interact with the medium and are expected to be sensitive to its energy density through the mechanism of parton energy loss. Due to the large mass of heavy quarks the suppression of small angle gluon radiation should reduce their energy loss, and consequently any suppression of heavy-quark mesons like $D$ and $B$ mesons at high- $p_{T}$ is expected to be smaller than that observed for hadrons consisting of light quarks [3]. 
We quantify the medium effects on high- $p_{\mathrm{T}}$ production in nucleus-nucleus collisions, $\mathrm{A}+\mathrm{A}$, with the nuclear modification factor which is defined as following:

$$
\mathrm{R}_{\mathrm{AA}}\left(\mathrm{p}_{\mathrm{T}}\right)=\frac{1}{\left\langle\mathrm{~N}_{\text {coll }}\right\rangle} \times \frac{\text { yield per } \mathrm{A}+\mathrm{A} \text { collision }}{\text { yield per } p+p \text { collision }}=\frac{1}{\left\langle\mathrm{~N}_{\text {coll }}\right\rangle} \times \frac{\mathrm{d}^{2} \mathrm{~N}^{\mathrm{A}+\mathrm{A}} / \mathrm{dydp}_{\mathrm{T}}}{\mathrm{d}^{2} \mathrm{~N}^{p+p} / \mathrm{dydp}_{\mathrm{T}}}
$$

This factor reflects the deviation of measured distributions of nucleus-nucleus, $\mathrm{A}+\mathrm{A}$, transverse momentum, at given impact parameter $b$, from measured distributions of an incoherent superposition of nucleon-nucleon $(p+p)$ transverse momentum, scaled by the average number of expected binary collisions $\left\langle N_{\text {coll }}\right\rangle$. This normalization often is known as "binary collisions scaling". In the absence of any modifications due to the 'embedding' of elementary collisions in a nuclear collision, we expect $\mathrm{R}_{\mathrm{AA}}=1$ at high- $p_{\mathrm{T}}$. At low $p_{\mathrm{T}}$, where particle production follows a scaling with the number of participants, the above definition of $\mathrm{R}_{\mathrm{AA}}$ leads to $\mathrm{R}_{\mathrm{AA}}<1$ for $p_{\mathrm{T}}<2 \mathrm{GeV} / \mathrm{c}$.

However, the interpretation of heavy-ion collision data is complicated by the fact that heavy quark production is modified in a nuclear target by cold nuclear matter (CNM) effects. These include modification of the parton density functions in a nucleus, parton energy loss in $\mathrm{CMN}$, and (for quarkonia states), breakup due to collisions with nucleons in the target. To disentangle such effects, multiple measurements are needed, such as collisions of different ions and at different collision energies. RHIC has the unique ability to store and collide nuclei of different mass, i.e. $\mathrm{Au}+\mathrm{Au}, \mathrm{Cu}+\mathrm{Cu}$ and $U+U$, and also collide asymmetric systems such as $d+\mathrm{Au}, \mathrm{He}+\mathrm{Au}$ and $\mathrm{Cu}+\mathrm{Au}$. By observing the modification in more elementary systems like in $d+\mathrm{Au}$ and $\mathrm{He}+\mathrm{Au}$, one may hope to understand the initial modifications before the QGP is formed.

Heavy quark production has been studied by the PHENIX experiment at RHIC via measurements of single leptons from semi-leptonic decays in both the electron channel at mid-rapidity and in the muon channel at forward-rapidity. In this paper I will summarize the latest PHENIX results concerning open and closed heavy flavor production as a function of beam energy and systems size.

\section{PHENIX Experiment}

The PHENIX detector [4] comprises three separate spectrometers in three pseudorapidity $(\eta)$ ranges. Two central arms at midrapidity cover $|\eta|<0.35$ and have an azimuthal coverage $(\phi)$ of $\pi / 2$ rad each, while muon arms at backward and forward rapidity cover $-2.2<\eta<-1.2$ and $1.2<\eta<2.4$, respectively, with full azimuthal coverage. In the central arms, heavy quark production was studied via measurements of single leptons (electrons) from semi-leptonic decays. Charged particle tracks are reconstructed using the drift chamber and pad chambers. Electron candidates are selected by matching charged tracks to hits in the Ring Imaging Cherenkov (RICH) counters and clusters in the Electromagnetic Calorimeter (EMCal). At forward and backward rapidity, the heavy quark production is measured via dimuon decays. Muons are identified by matching tracks measured in cathode-strip chambers, referred to as the muon tracker (MuTr), to hits in alternating planes of Iarocci tubes and steel absorbers, referred to as the muon identifier (MuID). Each muon arm is located behind a thick copper and iron absorber that is meant to stop most hadrons produced during the collisions, so that the detected muons must penetrate 8 to 11 hadronic interaction lengths of material in total. Beam interactions are selected with a minimum-bias (MB) trigger requiring at least one hit in each of the two beam-beam counters (BBCs) located at positive and negative pseudorapidity $3<|\eta|<3.9$.

\section{Open heavy flavor - single electron/muon measurements}

Open heavy flavor production is measured in PHENIX through the measurement of inclusive electrons or muons $[5,6]$. These analyses use a cocktail method to remove fake and real electrons/muons from 


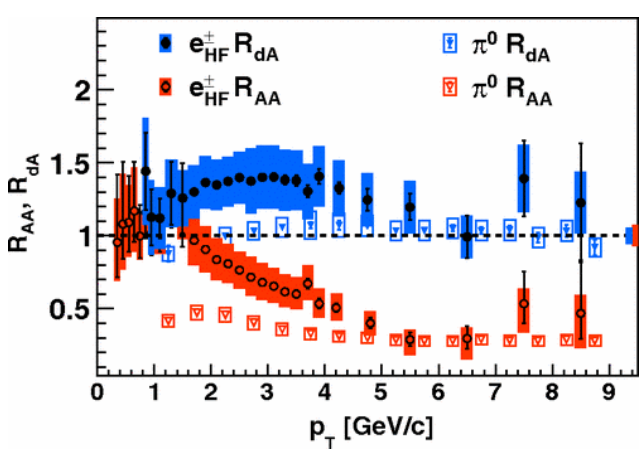

(a) $e_{H F}^{ \pm}$and $\pi^{0}: \mathrm{Au}+\mathrm{Au}$ vs. $d+\mathrm{Au}$ at $\sqrt{s_{N N}}=200 \mathrm{GeV}$

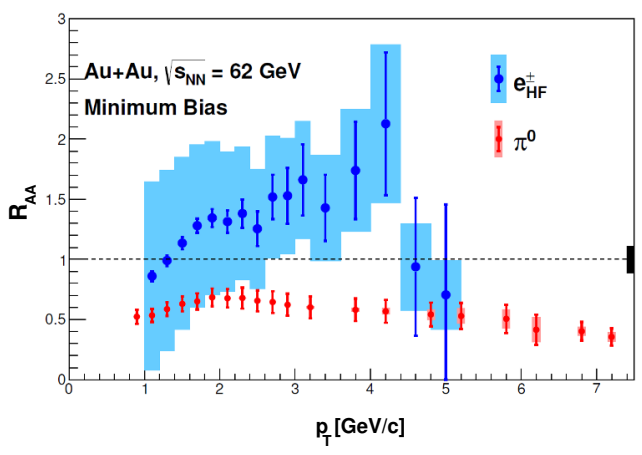

(b) $e_{H F}^{ \pm}$and $\pi^{0}: \mathrm{Cu}+\mathrm{Cu}$ at $\sqrt{s_{N N}}=62.4 \mathrm{GeV}$

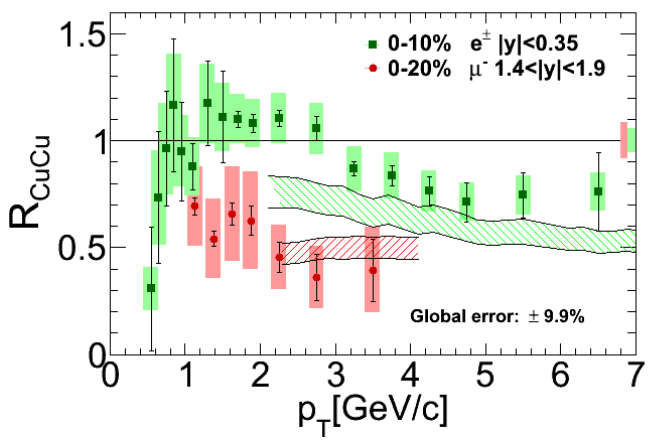

(c) $e_{H F}^{ \pm}$and $\mu_{H F}^{-}: \mathrm{Cu}+\mathrm{Cu}$ at $\sqrt{s_{N N}}=62.4 \mathrm{GeV}$

Figure 1. The nuclear modification factors $R_{d A u}\left(p_{\mathrm{T}}\right)$ and $R_{A A}\left(p_{\mathrm{T}}\right)$ for the $\pi^{0}, e_{H F}^{ \pm}$and $\mu_{H F}^{-}$: panels (a) and (b) for minimum bias $d+\mathrm{Au}, \mathrm{Au}+\mathrm{Au}$ and panel (c) for central collisions $\mathrm{Cu}+\mathrm{Cu}$. The two boxes on the right side of the plot represent the global uncertainties in the $d+\mathrm{Au}, \mathrm{Au}+\mathrm{Au}(\mathrm{Cu}+\mathrm{Cu})$ values of $N_{\text {coll }}[7-15]$.

the data sample. i.e. for the electron measurement, the electrons that come from either meson decay or photon conversions are measured and subtracted from the inclusive spectrum and the remainder is attributed to electrons coming from the semi-leptonic decay of $D$ and $B$ mesons. This remainder is also referred to as the non-photonic electron (NPE) component. PHENIX has measured spectra of the single electrons [7-10] and single muons $[10,11]$ from heavy flavor in $p+p$ and $\mathrm{Cu}+\mathrm{Cu}$ collisions as well single electrons from heavy flavor in $\mathrm{Au}+\mathrm{Au}[12,13]$ and $\mathrm{d}+\mathrm{Au}[14,15]$ collisions.

The effects of CMN are expected to be present in the initial state of A + A collisions; however, this CNM enhancement is convolved with the suppressing effects of hot nuclear matter. Figure 1(a) shows $R_{d A u}\left(p_{\mathrm{T}}\right)$ and $R_{A A}\left(p_{\mathrm{T}}\right)$ for $e_{H F}^{ \pm}$and the neutral pion $\left(\pi^{0}\right)$, for which only small CNM effects are observed between $e_{H F}^{ \pm}$and $\pi^{0}$ for $p_{\mathrm{T}}<5 \mathrm{GeV} / \mathrm{c}$. Above $p_{\mathrm{T}} \approx 5 \mathrm{GeV} / \mathrm{c}$, where the CNM effects on both species, $e_{H F}^{ \pm}$and $\pi^{0}$, are small, their $R_{A A}\left(p_{\mathrm{T}}\right)$ values are consistent within uncertainties. However, in the range where CNM enhancement is large for $e_{H F}^{ \pm}$and small on $\pi^{0}$, the corresponding $e_{H F}^{ \pm} R_{A A}\left(p_{\mathrm{T}}\right)$ values are consistently above the $\pi^{0}$ values. This could suggest that the difference in the initial state cold nuclear matter effects due to the mass-dependent Cronin enhancement is reflected in the final state spectra of these particles in $\mathrm{Au}+\mathrm{Au}$ collisions, although alternate explanations involving massdependent partonic energy loss in the hot medium are not ruled out. 


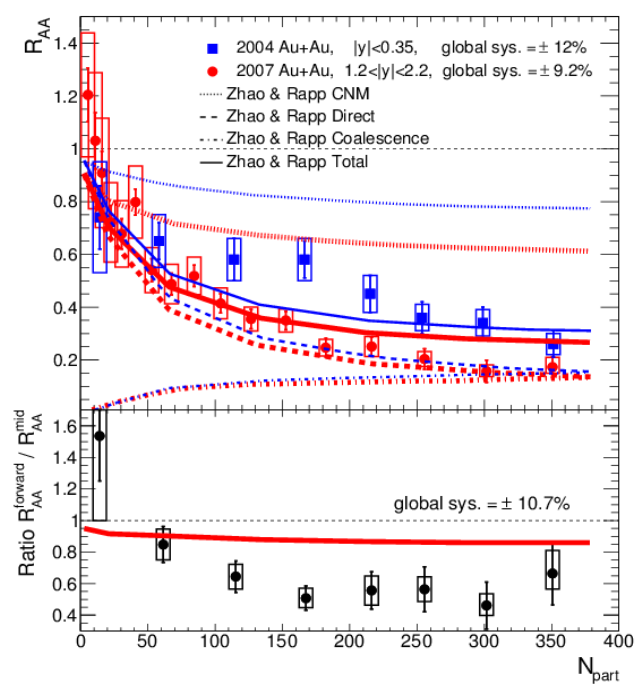

Figure 2. The upper panel shows the nuclear modification factor, $R_{A A}$ as a function of centrality. The lines show theoretical calculations: the effect of cold nuclear matter (dotted, uppermost pair), direct production (dashed), and coalescence (dot-dashed). The solid lines represent the sum of all effects considered. The lower panel shows the ratio of forward- to midrapidity $R_{A A}$ Ref. [19].

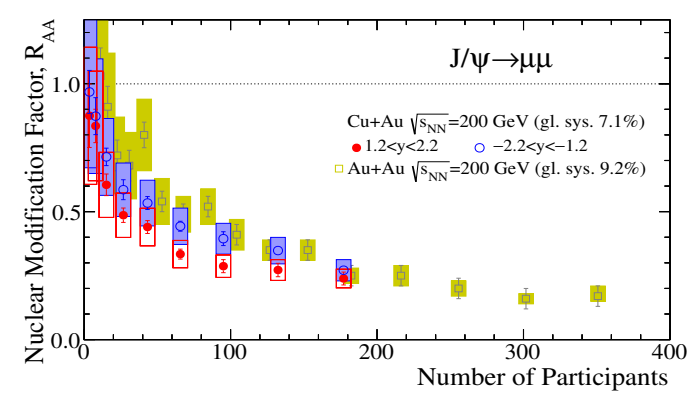

Figure 3. Nuclear modification factor, $R_{A A}$ as a function of centrality in $\mathrm{Cu}+\mathrm{Au}$ collisions at forward (red) and backward (blue) rapidity. For comparison, the $\mathrm{Au}+\mathrm{Au}$ (green) data are also shown, averaged over forward and backward rapidities Ref. [21].

To study the interplay between initial-state effects and final-states effects for heavy flavor $\left(e_{H F}^{ \pm}\right)$ productions, PHENIX measured the $R_{A A}\left(p_{\mathrm{T}}\right)$ in $\mathrm{Au}+\mathrm{Au}$ collisions at low beam energy, $\sqrt{s_{N N}}=$ $62.4 \mathrm{GeV}$. These $R_{A A}\left(p_{\mathrm{T}}\right)$ values for $e_{H F}^{ \pm}$in $\mathrm{Au}+\mathrm{Au}$ collisions at $62.4 \mathrm{GeV}$ are compared to $\pi^{0} R_{A A}\left(p_{\mathrm{T}}\right)$ at the same energy as shown in Figure 1(b). At $62.4 \mathrm{GeV}$, the competition, if present, favors heavyflavor enhancement over suppression. This is consistent with previous results with hadrons where the Cronin enhancement increases as the collision energy decreases [9].

PHENIX measurements of heavy flavor muons $\left(\mu_{H F}^{-}\right)$at forward rapidity $(1.4<\mathrm{y}<1.9)$ in central $\mathrm{Cu}+\mathrm{Cu}$ collisions at $\sqrt{s_{N N}}=62.4 \mathrm{GeV}$ show a significant suppression [9]. The magnitude of this suppression at forward-rapidity in $\mathrm{Cu}+\mathrm{Cu}$ (shown in Fig.1(c)) is compared to the suppression of $e_{H F}^{ \pm}$ in central $\mathrm{Au}+\mathrm{Au}$ collisions at mid-rapidity at the same energy. As open heavy flavor is significantly more suppressed at forward-rapidity than at mid-rapidity in $\mathrm{Cu}+\mathrm{Cu}$, additional nuclear effects, such as gluon shadowing at low-Björken- $x$ or partonic energy loss in the nucleus, may be significant. The heavy flavor $e_{H F}^{ \pm}$and $\mu_{H F}^{-}$are compared in Fig.1(c) to a theoretical prediction that combines the effects of partonic energy loss, energy loss from fragmentation and dissociation, and includes nuclear matter effects such as shadowing and Cronin enhancement due to parton scattering in the nucleus [16]. While consistent within uncertainties, the model predicts more suppression for heavy flavor electrons than seen in the data.

\section{Heavy quarkonia - $J / \psi$ production versus system size}

Heavy quarkonia has long been proposed as a sensitive probe of the color screening length and deconfinement in the quark-gluon plasma [17]. It was originally suggested by Matsui and Satz [18] that 


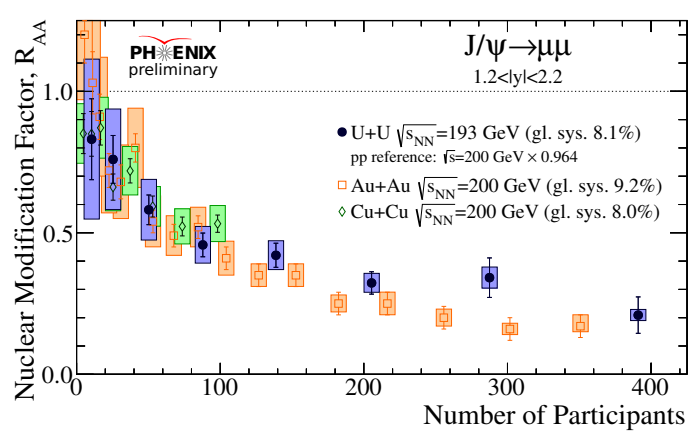

Figure 4. Nuclear modification factor, $R_{A A}\left(p_{\mathrm{T}}\right)$, measured in $U+U$ (blue) collisions at $\sqrt{s_{N N}}=193 \mathrm{GeV}$ as a function of centrality. For comparison, $\mathrm{Au}+\mathrm{Au}$ (red) and $\mathrm{Cu}+\mathrm{Cu}$ (green) data are also shown. The $U+U$ $R_{A A}\left(p_{\mathrm{T}}\right)$ results are preliminary.

a signature of the formation of the QGP would be that $J / \psi$ states that are initially bound may disassociate due to color screening in the high temperature QGP matter. The picture that was originally proposed is complicated by competing effects, which modify quarkonia production and survival in cold and hot nuclear matter. Heavy quarkonia measurements are made in PHENIX by either detecting opposite sign electrons at mid-rapidity $(|y|<0.35)$ or by detecting opposite sign muons at forwardrapidity $(1.2<|y|<2.2)$, reconstructing the invariant mass of the di-lepton pair, and subtracting the continuum background [11]. The versatility of RHIC to provide heavy ion collisions at different center of mass energies and for different ion species has been further demonstrated during RUN-12 by providing first $U+U$ and $\mathrm{Cu}+\mathrm{Au}$ collisions at $\sqrt{S_{N N}}=193$ and $200 \mathrm{GeV}$, respectively. Both systems serve as an important test of the initial geometry.

\section{1 $J / \psi$ in $\mathrm{Au}+\mathrm{Au}$ collisions}

The nuclear modification factor, $R_{A A}\left(p_{\mathrm{T}}\right)$, for $J / \psi$ as a function of centrality $\left(\mathrm{N}_{\text {part }}\right)$ at mid-rapidity and forward-rapidity from $\mathrm{Au}+\mathrm{Au}$ collisions at $\sqrt{s_{N N}}=200 \mathrm{GeV}$ is shown in Fig. 2. The data show that the suppression of $J / \psi$ at forward-rapidity is stronger than at mid-rapidity, even though the energy density is expected to be slightly larger at mid-rapidity. This could be due to stronger cold nuclear matter effects at forward-rapidity, or possibly stronger coalescence of charm quarks at hadronization at mid-rapidity, or both.

The comparison of the experimental data to the most recent theoretical calculations that incorporate a variety of physics mechanisms including gluon saturation, gluon shadowing, initial-state parton energy loss, cold nuclear matter breakup, color screening, and charm recombination are presented on Fig. 2 and described in details by PHENIX in Ref. [19].

\section{2 $J / \psi$ in $\mathrm{Cu}+$ Au collisions}

Hot matter effects and CNM effects are present together in heavy ion collisions, and both are important. In $\mathrm{Au}+\mathrm{Au}$ collisions at RHIC, for example, the addition of hot matter effects increases the suppression of the $J / \psi$ by a factor of roughly two over what would be expected if only CNM effects were present $[19,20]$. By colliding ions different sizes, like $\mathrm{Cu}+\mathrm{Au}$, the cold nuclear matter effects is expected to be different at forward and backward rapidities. The comparison of $d+A u, A u+A u$ and 
$\mathrm{Cu}+\mathrm{Au} J / \psi$ modifications across rapidities may provide key insight on the balance of cold and hot nuclear matter effects, and whether they are factorizable.

Figure 3 shows nuclear modification factor, $R_{A A}\left(p_{\mathrm{T}}\right)$, for $J / \psi$ as a function of $\mathrm{N}_{\text {part }}$ for $\mathrm{Cu}+\mathrm{Au}$ collisions at $\sqrt{s_{N N}}=200 \mathrm{GeV}$ [21]. The $R_{A A}\left(p_{\mathrm{T}}\right)$ for $\mathrm{Au}+\mathrm{Au}$ collisions at the same collision energy and rapidity is shown in Fig. 3 for comparison. The dependence of the $\mathrm{Cu}+\mathrm{Au}$ nuclear modification on $\mathrm{N}_{\text {part }}$ at backward (Au-going) rapidity is similar to that for $\mathrm{Au}+\mathrm{Au}$ collisions, while the $\mathrm{Cu}+\mathrm{Au}$ $R_{A A}\left(p_{\mathrm{T}}\right)$ at forward (Cu-going) rapidity is little lower.

\section{3 $J / \psi$ in $U+U$ collisions}

The study of collisions of deformed nuclei like ${ }^{238} \mathrm{U}$ was initially proposed [22] because they promise an additional gain of initial energy density relative to collisions of spherical nuclei. A significantly deformed initial geometry at very high energy density for specific orientations of $U+U$ collisions is expected to have observable effects on elliptic fow, jet quenching, $J / \psi$ suppression and other observables that characterize the properties of the quark gluon plasma (QGP) [22, 23]. The interesting configurations with high energy density can be selected in experiments by using a combined cut on the elliptic flow value and the number of spectators for high multiplicity events [24].

The nuclear modification factor, $R_{A A}\left(p_{\mathrm{T}}\right)$, for $J / \psi$ as a function of centrality, $\mathrm{N}_{\text {part }}$, at forwardrapidity $(1.2<|\mathrm{y}|<2.2)$ from $U+U$ collisions is shown in Fig. 4. The $R_{A A}\left(p_{\mathrm{T}}\right)$ for $\mathrm{Au}+\mathrm{Au}$ and $\mathrm{Cu}+\mathrm{Cu}$ collisions at similar energy, $\sqrt{s_{N N}}=200 \mathrm{GeV}$, and rapidity are also shown in Fig 4 for comparison. The centrality dependence of $U+U R_{A A}\left(p_{\mathrm{T}}\right)$ for $J / \psi$ shows a similar trend to the lighter systems, $\mathrm{Au}+\mathrm{Au}$ and $\mathrm{Cu}+\mathrm{Cu}$.

\section{Summary}

PHENIX has shown first measurements at RHIC from two very unique systems: $U+U$ and $\mathrm{Cu}+\mathrm{Au}$ at $\sqrt{S_{N N}}=193$ and $200 \mathrm{GeV}$, respectively. Data from both systems were compared to $\mathrm{Au}+\mathrm{Au}$ and $\mathrm{Cu}+\mathrm{Cu}$ collisions at similar energy, $\sqrt{S_{N N}}=200 \mathrm{GeV}$. The comparison aimed to shed more light on the role of the initial collision configuration and the final data observables. The $U+U$ system holds the highest energy density so far measured at RHIC. We observed that the dependence of the $\mathrm{Cu}+\mathrm{Au}$ nuclear modification for $J / \psi$ as function of centrality, $\mathrm{N}_{\text {part }}$, at backward (Au-going) rapidity is similar to that for $\mathrm{Au}+\mathrm{Au}$ collisions, while the $\mathrm{Cu}+\mathrm{Au} R_{A A}\left(p_{\mathrm{T}}\right)$ at forward $(\mathrm{Cu}$-going) rapidity is noticeably lower. Moreover, the centrality dependence of $U+U R_{A A}\left(p_{\mathrm{T}}\right)$ for $J / \psi$ shows a similar trend to the lighter systems, $\mathrm{Au}+\mathrm{Au}$ and $\mathrm{Cu}+\mathrm{Cu}$ at similar energy. These observation lead to the conclusion that the centrality dependence of $R_{A A}\left(p_{\mathrm{T}}\right)$ for $J / \psi$ at given energy is less sensitive to the system size. Therefore these new PHENIX results of $R_{A A}\left(p_{\mathrm{T}}\right)$ for $J / \psi$ in $U+U$ and $\mathrm{Cu}+\mathrm{Au}$ collisions are crucial for testing different theoretical approaches.

\section{References}

[1] I. Arsene et al., Nucl. Phys. A 757, 1 (2005); B. B. Back et al., Nucl. Phys. A 757, 28 (2005);

J. Adams et al., Nucl. Phys. A 757, 102 (2005); K. Adcox et al., Nucl. Phys. A, 757, 184 (2005).

[2] Z. Lin and M. Gyulassy, Phys Rev. C 51, 2177 (1995).

[3] Y. L. Dokshitzer and D. E. Kharzeev, Phys. Lett. B 519, 199 (2001).

[4] K. Adcox et al., Nucl. Instrum. Methods A 99, 469 (2003).

[5] A. Adare et al., Phys. Rev. Lett. 112, 252301 (2014).

[6] A. Adare et al., Phys. Rev. Lett. 109, 242301 (2012). 
[7] A. Adare et al., Phys. Rev. Lett. 97, 252002 (2006).

[8] A. Adare et al., Phys. Rev. Lett. 103082002 (2009).

[9] A. Adare et al., Phys. Rev. C 86024909 (2012).

[10] A. Adare et al., Phys. Rev. C 90, 034903 (2014).

[11] A. Adare et al., Phys. Rev. D 76, 092002 (2007).

[12] B.I. Abelev et al., Phys. Lett. B 655, 104 (2007); S.S. Adler et al., Phys. Rev. Lett. 98, 172302 (2007); S.S. Adler et al., Phys. Rev. Lett. 96, 202301 (2006); S.S. Adler et al., Phys. Rev. Lett. 91, 072303 (2003).

[13] R. Nouicer et al., Nuclear Physics A 862, 64 (2011).

[14] A. Adare et al., Phys. Rev. Lett. 109, 242301 (2012).

[15] R. Nouicer et al., Journal of Physics : Conference Series 420, 012021 (2013).

[16] R. Sharma, I. Vitev, and B.-W. Zhang, Phys. Rev. C 80, 054902 (2009).

[17] B. Muller, Nucl. Phys. A 750, 84 (2005).

[18] T. Matsui and H. Satz, Phys. Lett. B 78, 416 (1986).

[19] A. Adare et al., Phys. Rev. C 84, 054912 (2011).

[20] N. Brambilla, S. Eidelman, B. K. Heltsley, R. Vogt, et al., Eur. Phys. J. C 71, 1534 (2011).

[21] A. Adare et al, arXiv:1404.1873

[22] E. V. Shuryak, Phys.Rev., C 61, 034905 (2000).

[23] U. W. Heinz and A. Kuhlman, Phys. Rev. Lett. 94, 132301 (2005).

[24] A. J. Kuhlman and U. W. Heinz, Phys.Rev., C 72, 037901 (2005). 